\title{
Amnion-Chorion Strips in the Treatment of Peri-Implantitis, Report of a Case
}

\author{
Mohamed A Maksoud, DMD*
}

Oral Medicine Infection and Immunity, Harvard University School of Dental Medicine, USA

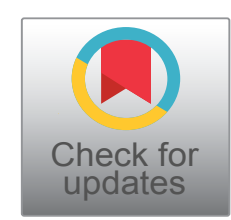

*Corresponding author: Mohamed A Maksoud, Oral Medicine Infection and Immunity, Harvard University School of Dental Medicine, 188 Longwood Avenue, Boston, Ma., 02115, USA

\begin{abstract}
Dental implant disease described as Peri-implantitis is bacterial inflammation of the implant surrounded soft and hard tissue that can lead to bone loss and eventual failure of the implant fixture. Several protocols have been introduced to treat implantitis including the use of systemic and local antibiotics in addition to bone grafting in advanced cases. Although the treatment outcome is effective in the early to moderate cases its unpredictable in cases with severe bone loss. Human placental tissue has been introduced in medicine for the treatment of burns and ophthalmic disease around the turn of the century. In dentistry, the placental derived tissue has demonstrated a promising and effective role in the treatment of soft and hard tissue deficiencies within the oral cavity. In this report, the Amnion-Chorion membrane was used in strips and mixed with the bone graft material after treatment of the contaminated implant surface. The results showed substantial bone fill around the implant with uneventful healing of the surrounding mucosal tissue. This technique can serve as a predictable protocol for the treatment of advanced dental implant disease.
\end{abstract}

\section{Keywords}

Dental implants, Implant disease, Inflammation

\section{Background}

Membranes derived from the human placentas have already been used in the field of medicine for skin grafts, treatment of burns, and ulcerated skin conditions. Recently, placenta derived membranes have been used in dentistry for root coverage procedure. The use of such allografts is based on the hypothesis that the unique inherent biologic properties in placenta enhance wound healing and may even propagate regeneration. In periimplantitis where there is partial loss of the osseintegrated implant in addition to pocket formation the challenge is to reestablish the bone to implant bond using a bone graft. All procedures introduced to clinical implantology are not superior to others with no predictable outcome.

\section{Case Presentation}

A 41-years-old patient presented with a restored dental implant in the mandibular posterior molar area, the implant fixture was placed approximately eight years ago and restored few months afterwards. The intra-oral exam revealed 5-6 $\mathrm{mm}$ pockets around the implant with slight inflammation of the surrounding soft tissue including bleeding upon probing and exudate. No mobility was detected and the patient

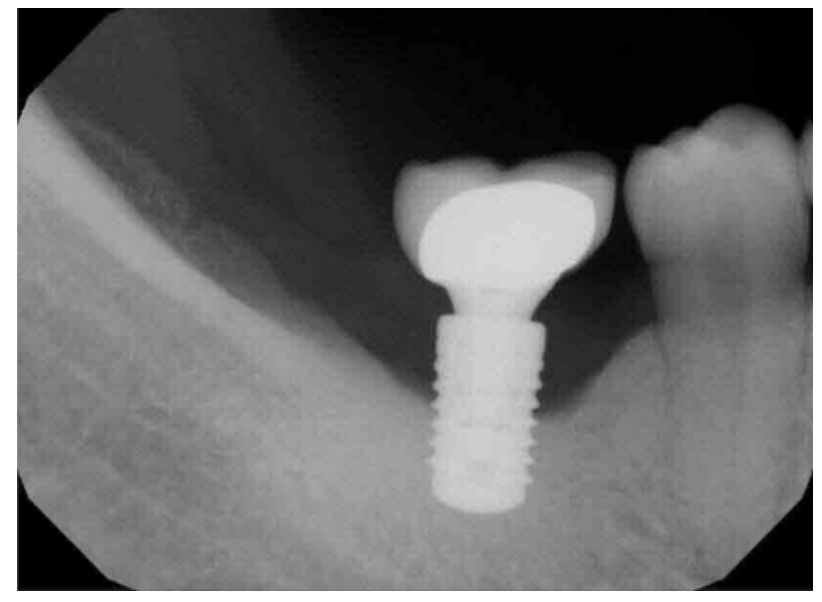

Figure 1: Periapical film of the implant before treatment. 


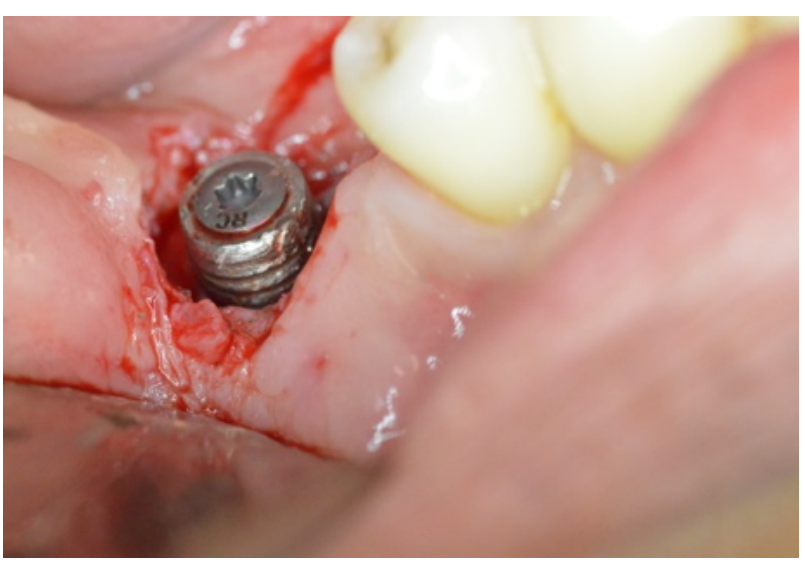

Figure 2: Circumferential incision around the implant with removal of the granulation tissue.

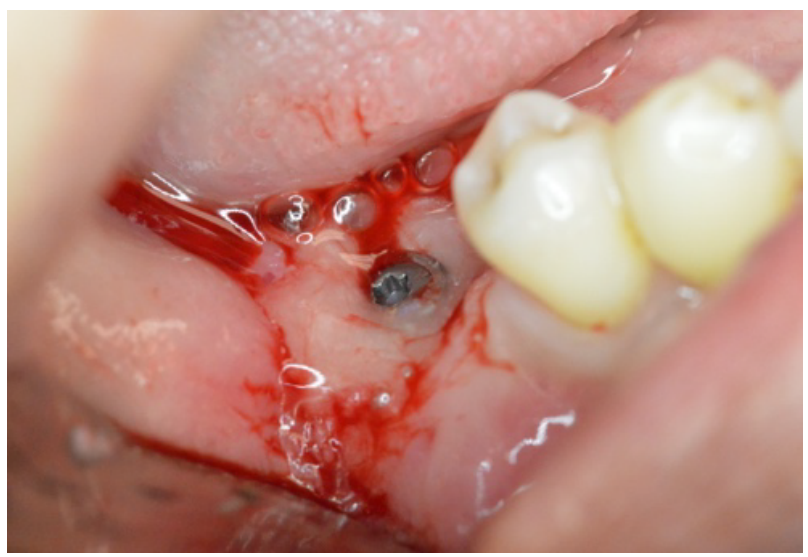

Figure 3: Prefgel placed around the implant following ultrasonic debridement.

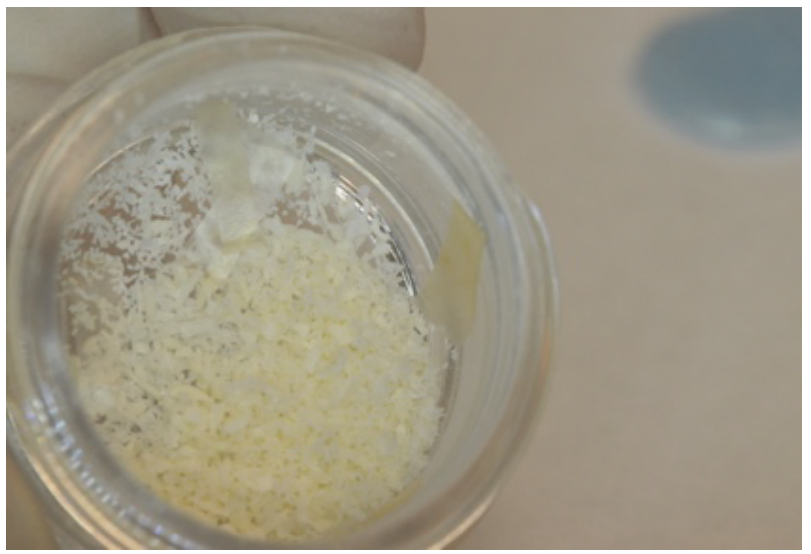

Figure 4: The allograft bone mixed with the amnio strips.

was asymptomatic. The radiographic exam (Figure 1) revealed considerable bone loss with no other findings. The implant crown was removed and a cover screw placed. The Surgical treatment included a circumferential incision around the implant (Figure 2) followed by excavating the granulation tissue into the bony defect, scaling the implant surface using ultrasonic scalers and then using Prefgel to decontaminate the implant surface (Figure 3). A bone mix of mineralized cortico-cancellous allograft with Am-

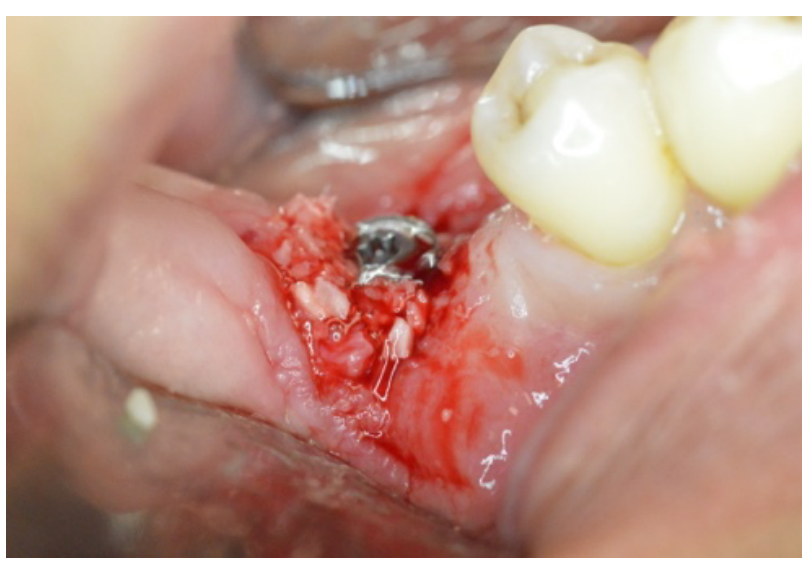

Figure 5: The allograft mix placed around the implant.

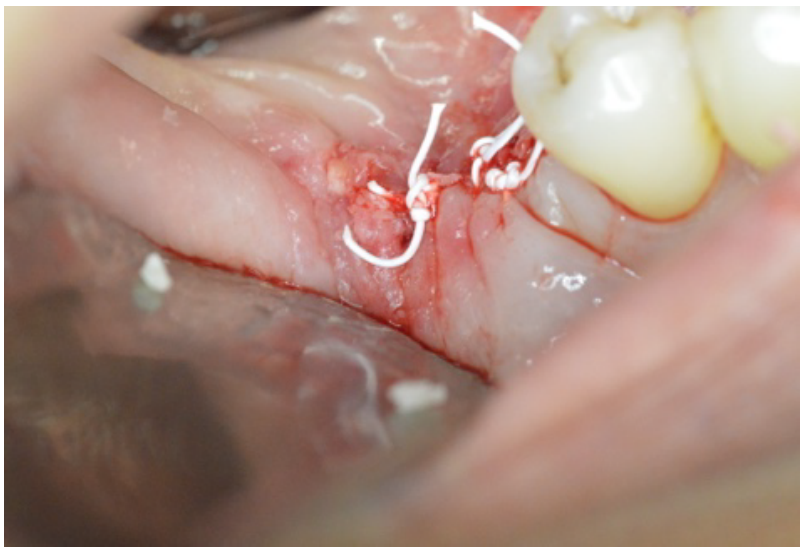

Figure 6: Suturing.

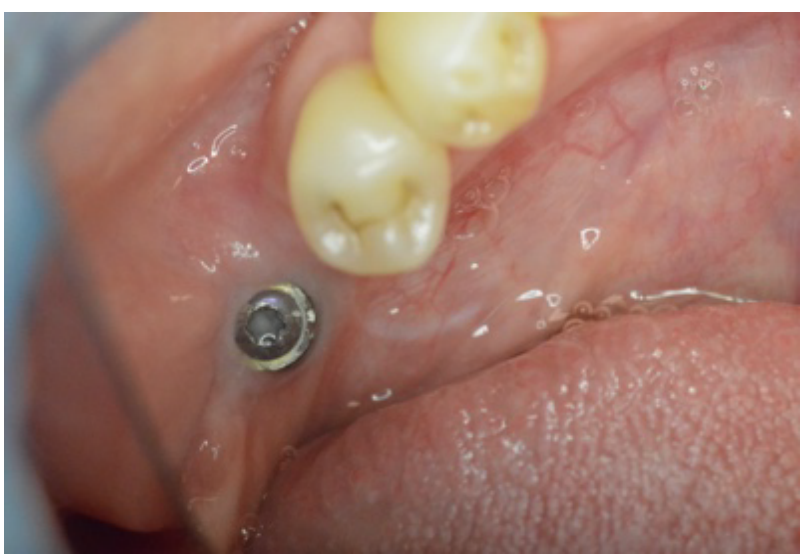

Figure 7: Two weeks healing following the procedure.

nion-Chorion strips used to fill the bone defect (Figure 4 and Figure 5) and followed by mono filament non-resorbable suture (Figure 6). The patient placed on systemic antibiotics for one week and was prescribed chlorhexidine mouth wash in addition to mild analgesics. Two weeks post-operatively the patient presented with uneventful healing of the soft tissue and complete healing of the surgical wound over the bone graft mass (Figure 7). Three months following the procedure a periapical film showed considerable fill of the bone defect with integration to the implant surface (Figure 8). 


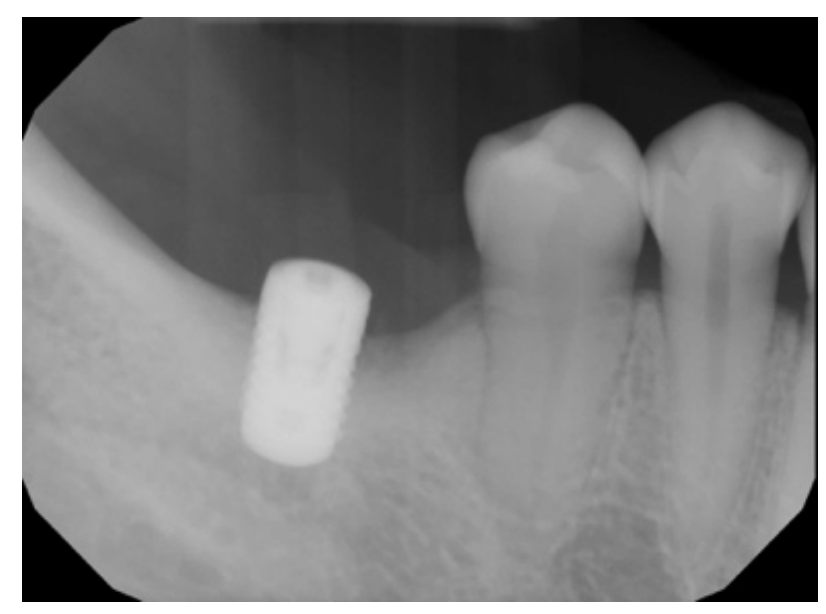

Figure 8: Periapical film of the treated implant eight weeks following the treatment.

\section{Discussion}

Treatment of periimplantitis is one on the challenging procedure in implant dentistry in which bone re integration to the infected implant surface is unpredictable. The introduction of Amniotic membrane into medicine and dentistry with its abundant growth factors has shown promising results when used over bone grafts. Membranes derived from the human placentas have already been used in the field of medicine for skin grafts, treatment of burns, and ulcerated skin conditions. The use of such allografts is based on the hypothesis that the unique inherent biologic properties in placenta enhance wound healing and may even propagate regeneration. The use of the same membrane when cut into strips and mixed with the bone allograft mass has not been clinically reported yet. The main objective is to expedite the process of osteogenesis on the implanted bone allograft [1-5].

\section{Conclusion}

In this case, the predictable outcome could be due to the aggressive role of the Amnio strips into the bone to reestablish osseointegration. In addition, the soft tissue healing of the wound was progressive in two weeks with no exposure of the bone graft. Further studies are encouraged with histological investigation to determine the quality of the newly formed bone around the implant surface.

\section{References}

1. Levin B, Rubinstein S, Rosenthaler H, Fujiki T, Tawil P (2013) Advanced surgical and restorative therapies aimed at rehabilitation of a severe dentoalveolar defect in the esthetic zone. J Imp Adv Clin Dent 5: 17-27.

2. Maksoud M, Guze K (2018) Tissue expansion of dental extraction sockets using dehydrated human amnion/chorion membrane: Case series. Clin Adv Periodontics 8: 111-114.

3. Froum S, Rosen P (2014) Reentry evaluation following treatment of peri-implantitis with a regenerative approach Int J Periodontics Restorative Dent 34: 47-59.

4. Holtzclaw D, Toscano N (2011) BioXclude ${ }^{\circledR}$ placental allograft tissue membrane in combination with bone allograft for site preservation: A case series. J Imp Adv Clin Dent 3: $35-50$.

5. Wallace W, Cobb C (2011) Histological and computed tomography analysis of amnion chorion membrane in guided bone regeneration in socket augmentation. J Imp Adv Clin Dent 3: 61-72. 